\title{
Dbrane Phase Transitions and Monodromy in $K$-theory
}

\author{
Sergei Gukov* and Vipul Periwal \\ Department of Physics, Princeton University, Princeton, New Jersey 08544
}

(PUPT-1884; ITEP-TH-39/99; hep-th/9908166)

\begin{abstract}
Majumder and Sen have given an explicit construction of a first order phase transition in a non-supersymmetric system of Dbranes that occurs when the $B$ field is varied. We show that the description of this transition in terms of $K$-theory involves a bundle of $K$ groups of non-commutative algebras over the Kähler cone with nontrivial monodromy. Thus the study of monodromy in $K$ groups associated with quantized algebras can be used to predict the phase structure of systems of (non-supersymmetric) Dbranes.
\end{abstract}

A major development in string theory over the past couple of years has been an increasing understanding of non-supersymmetric Dbranes and their dynamics, initiated largely by Sen [1]. The relevance of $K$-theory for the classification of Dbrane charges was pointed out by Witten [2] following earlier work of Minasian and Moore [3]. Reviews of these developments are $[$ 田圂].

The motivation for the present work was an intriguing observation made by Majumder and Sen [7]. Using an exact conformal field theory description [8] of non-supersymmetric Dbranes on a K3 surface, they found three different phases in the region on the moduli space where the surface looks like an orbifold $X=T^{4} / \mathbb{Z}_{2}$. The phase diagram in Fig. 1 represents different states (labeled as $I, I I$ and $I I I$ ) of two D-branes f wrapped over exceptional 2cycles $S_{1}$ and $S_{2}$ in $X$, which correspond to fixed points in the orbifold limit. In phase $I$ the two Dbranes recombine into a single Dbrane wrapped over a non-supersymmetric cycle with the homology class $[S]=\left[S_{1}\right]+\left[S_{2}\right]$. This happens when the minimal radius of $T^{4}$ becomes less than the difference of the $B$-field fluxes through $S_{1}$ and $S_{2}, \zeta<R$ with the appropriate normalization. From the analysis of the tachyon potential [7]

$$
V(\alpha) \propto\left(\frac{1}{4}\left(R_{c}-R\right) \cos (\alpha \pi)+\zeta \cos \left(\frac{1}{2} \alpha \pi\right)\right)
$$

\footnotetext{
${ }^{*}$ On leave from the Institute of Theoretical and Experimental Physics and the L. D. Landau Institute for Theoretical Physics.

1 The spectrum of $\mathrm{D} p$-branes in Type II string theory is periodic under $p \rightarrow p+2$, so the value of $p$ will not be important in our discussion, as long as $p \geq 2$. In $K$-theory this periodicity is just Bott periodicity.
} 
( $\alpha$ is the parameter labelling the marginal deformation, normalized so that $\alpha=0 \bmod 2$ represents the pair of Dbranes and $\alpha=1 \bmod 2$ represents the non-BPS brane) one can see that transitions between regions $I$ and $I I$ and between regions $I$ and $I I I$ are second order. There is no discontinuous jump in the location of the minimum of the tachyon potential in these transitions. On the other hand, as we pass through the phase boundary between regions $I I$ and $I I I$ the Dbranes flip their orientation via a first order phase transition. The three phases coexist at the critical radius $R_{c}(\zeta=0)$ where the anti-periodic tachyonic mode becomes exactly marginal.

We want to understand this phase diagram from the point of view of $K$-theory and see if such transitions are possible at all. $K$ groups classify Dbrane charges [3,2]. Therefore, this phase diagram indicates that a certain element of a $K$ group is mapped to an appropriate inverse as one crosses the transition line between phases $I I$ and $I I I$. In other words, it means that a $K$-group element undergoes a monodromy as one goes around the point $R_{c}$ in the $R-\zeta$ plane. Indeed, because there is no discontinuity along the lines of the second order phase transitions between phases $I I$ and $I$, and between phases $I$ and $I I I$, we do not expect to see these lines in the deformation of the $K$ groups. What this means precisely, and what can be predicted from this point of view, constitute the topics of the present paper.

As we explain below, the observation of Majumder and Sen is a rather general phenomenon in $K$-theory, and we will formulate the explicit condition when it takes place for a general space $X$. It will turn out that the appropriate setting for the analysis is algebraic $K$-theory (for introductory treatments see [9,10]), i.e. instead of a space $X$ we consider the ring (algebra) $\mathcal{A}$ of continuous functions on $X$. Although the algebraic $K$-theory of $\mathcal{A}$ is isomorphic to topological $K$-theory of $X$ for smooth $X$, the former has two major benefits:

- Even if the geometry of $X$ is singular, $\mathcal{A}$ may remain a well-defined algebra. For example, if $X$ is a quotient space with possible fixed point singularities, $\mathcal{A}$ is a crossed product algebra [12].

- Majumder and Sen consider a 'blow-up' of $X$ corresponding to a $B$-field flux through 2-cycles in $X$. Such non-geometric 'defomations' of $X$ have a natural interpretation in terms of non-commutative deformations of $\mathcal{A}[11] 13$.

An attempt to formulate string theory in algebraic terms can be found in [14].

We briefly recall the definition of $K$-groups that measure Dbrane charges. In topological $K$-theory $K^{0}(X)$ is defined as the group of pairs $(E, F)$ of vector bundles modulo the equivalence relation $(E, F) \sim\left(E \oplus H, F \oplus H^{\prime}\right)$ which allows creation and annihilation of brane-anti-brane pairs with isomorphic gauge bundles $H$ and $H^{\prime}$. In algebraic $K$-theory the Grothendieck group $K_{0}(\mathcal{A})$ is defined in a similar way with "bundles over $X$ " replaced by "projective modules over $\mathcal{A}$ ". In practice, however, it is convenient to use another (equivalent) definition of $K_{0}(\mathcal{A})$ via idempotents in $M_{n}(\mathcal{A})$, the set of $n \times n$ matrices, with coefficients in $\mathcal{A}$. To allow direct sum and tensor product of idempotents, one actually has to consider the direct limit $M_{\infty}(\mathcal{A})=\lim M_{n}(\mathcal{A})$ with the inductive limit topology. Then unitary equivalence classes of projection operators $\alpha$ :

$$
\alpha * \alpha=\alpha
$$

in $M_{\infty}(\mathcal{A})$ form a semigroup $S \equiv S(\mathcal{A})$ under addition. The Grothendieck group associated with this semigroup is constructed as follows. On $S \times S$ define an equivalence relation $(a, b) \sim$ 
$\left(a^{\prime}, b^{\prime}\right)$ if $a+b^{\prime}=a^{\prime}+b$. Then $K_{0}(\mathcal{A})=S \times S / \sim$. By definition $K_{0}$ is a covariant functor, i.e. any homomorphism $\phi: \mathcal{A} \rightarrow \mathcal{B}$ of $C^{*}$-algebras $\mathcal{A}$ and $\mathcal{B}$ induces a homomorphism $\phi_{*}: K_{0}(\mathcal{A}) \rightarrow K_{0}(\mathcal{B})$. If we define a suspension as the set of continuous functions from the real line to $\mathcal{A}, \Sigma \mathcal{A} \equiv C(\mathbb{R} \rightarrow \mathcal{A}) \cong \mathcal{A} \otimes C(\mathbb{R})$, we may introduce the higher K-group $K_{1}(\mathcal{A}) \cong K_{0}(\Sigma \mathcal{A})$. Equivalently, one can define $K_{1}(\mathcal{A})$ as the abelian group of equivalence classes of invertible elements in $M_{\infty}(\mathcal{A})$, with equivalence defined by right translation by elements of the group that are in the identity component. Complex Bott periodicity is the statement that $K_{0}\left(\Sigma^{2} \mathcal{A}\right)=K_{0}(\mathcal{A})$.

According to Connes, Douglas and Schwarz [11], the presence of a uniform $B$-field can be interpreted in terms of a quantization of the function algebra of the manifold, à la Fedosov [15]. Their deep insight has led to numerous developments [12,13] on this theme. We are interested in a $B$-field which is not uniform [7]. Since no precise relation to deformation quantization has been established for this case, we assume that a general $B$-field leads to the deformed algebra $\mathcal{A}$ with the $*$ product defined by Kontsevich [16]. In fact, as it will become clear in a moment, the results of this paper do not depend on this assumption; one just has to know that turning on a $B$-field leads to some associative deformation of a product on $\mathcal{A}$ :

$$
f *_{\tau} g=f g+\tau f *_{1} g+\ldots
$$

where $\tau$ is the deformation parameter. In the following we will only make the $*$ symbol explicit when we need to emphasize the deformed product. The $*$ product depends not just on $B$ but also on the Kähler form $J$. However, this dependence is such that when $B=0$ the algebra is not expected to undergo any deformation [11, 13]. This is perhaps surprising at first sight since the natural variable in string theory is $B+i J$, but it follows from the modular-like invariance of the $*$ product under $T$ duality.

Thus, over each point in the $(R, \zeta)$ plane we have an algebra in which the product depends on the value of $z \equiv(R, \zeta)$. We shall take the undeformed algebra in our model to be the algebra at $z=R_{c}$. Therefore we define the deformation parameter $\tau \equiv z-R_{c}$. Computing the $K$ groups of these algebras at each point in the $z$-plane, we obtain a bundle of $K$ groups. These $K$ groups at different points on the $(R, \zeta)$ plane a priori have little to do with each other.

However, the important point is that $K$ groups are, generally speaking, rigid under deformation quantization [17]. This fact is the conceptual reason why BPS states survive turning on a $B$-field and why BPS conditions in the presence of a $B$-field are very simple to state in terms of the $*$ product [13]. Now it is easy to see the deformation of (2) under quantization. Given a projection $p$ in $\mathcal{A}_{0}$, where $\mathcal{A}_{0}$ is the algebra before quantization, we need to find a projection $p_{\tau}$ in $\mathcal{A}_{\tau}$ such that $p_{\tau} *_{\tau} p_{\tau}=p_{\tau}$ and $\lim _{\tau \rightarrow 0} p_{\tau}=p$. This can be solved recursively as a power series, or by using the differential equation

$$
p_{\tau}^{\prime} *_{\tau} p_{\tau}+p_{\tau} *_{\tau} p_{\tau}^{\prime}+p_{\tau} \Delta *_{\tau} p_{\tau}=p_{\tau}^{\prime},
$$

where $\Delta *_{\tau}$ denotes the derivative of the $\tau$ dependence in the $*$ product, with the obvious boundary condition at $\tau=0$. Thus there is a natural manner in which the fibres of the $K$-group bundle are all isomorphic, so it actually is a fibre bundle. Given this isomorphism, we can meaningfully consider the monodromy of sections of this fibre bundle as we move around $\tau=0$. 
In supersymmetric cases such monodromies are very well understood from the relation between the Mukai vector [11]:

$$
\vec{Q}=\operatorname{ch}(E) e^{-\frac{[B]}{2 \pi i}} \in H^{\text {even }}(X, \mathbb{Z})
$$

and the Chern character of a projective module $E$. In physics this expression appears in the Chern-Simons coupling of Ramond-Ramond fields. Let us explain the origin of the monodromy in algebraic $K$-theory by a simple example of Type IIB compactification on a non-commutative torus. An ordinary torus $T^{2}$ can be represented as a quotient space $X=S^{1} \times \mathbb{R} / \mathbb{Z}$. The corresponding crossed product algebra $\mathcal{A}_{0}=C\left(S^{1}\right) \times \mathbb{Z}$ is generated by two unitary operators that commute with each other. Its non-commutative deformation $\mathcal{A}_{\tau}=C\left(S^{1}\right) \times{ }_{\tau} \mathbb{Z}$ is defined by 'twisting' the multiplication by a $\tau \in \operatorname{Aut}\left(C\left(S^{1}\right)\right): a g=g \tau(a)$ for all $a \in C\left(S^{1}\right)$ and $g \in \mathbb{Z}$. The deformed algebra $\mathcal{A}_{\tau}$ is Morita equivalent to a noncommutative torus which is believed to describe a compactification on a torus with a $B$-field flux [11. Because $\mathcal{A}_{\tau}$ is still a crossed product algebra, we can use the Pimsner-Voiculescu exact sequence [18] to compute its $K$ groups:

$$
\ldots \longrightarrow K_{0}\left(C\left(S^{1}\right)\right) \stackrel{\mathrm{Id}-\tau}{\longrightarrow} K_{0}\left(C\left(S^{1}\right)\right) \longrightarrow K_{0}\left(C\left(S^{1}\right) \times_{\tau} \mathbb{Z}\right) \longrightarrow K_{1}\left(C\left(S^{1}\right)\right) \longrightarrow \ldots
$$

First of all, from this sequence we see the result, alluded to above, that $K$-theory is rigid under deformation quantization. This follows from the fact that the map $\mathrm{Id}-\tau$ is homotopic to zero, so that the Pimsner-Voiculescu exact sequence reduces to a short exact sequence and splits. The same statement is also true in real $K$-theory [19]. Furthermore, from (6) we find $K_{0}\left(\mathcal{A}_{\tau}\right)=\mathbb{Z}+\tau \mathbb{Z}$, so that charges of odd-dimensional Dbranes undergo a monodromy:

$$
\vec{Q} \longrightarrow\left(\begin{array}{ll}
1 & 1 \\
0 & 1
\end{array}\right) \vec{Q}
$$

as the $B$-field changes by a period.

In the non-supersymmetric situation considered by Majumder and Sen [7], we expect monodromy in going around the point $R_{c}$ in the $z$ plane. We wish to stress here that in the moduli space of K3 with a $B$-field this monodromy does not correspond to any non-trivial element of the first fundamental group. We do not have a sufficiently detailed understanding of the $K$ group bundle in the Majumder-Sen case to compute this monodromy exactly. However, we shall now explicitly construct a simple local model showing how such monodromy arises.

If $\alpha$ is the projection corresponding to phase $I I$, in going around a small loop centered at $R_{c}$ we expect to find a projection associated with the non-BPS Dbrane with the opposite charge. What is the projection associated with such an oppositely charged Dbrane? The sum of a charge and the opposite charge should be equivalent to a trivial bundle, but $-\alpha$ is not a projection so we have to work a little harder to find an inverse. Given a projection $\alpha$, and a projection $\pi_{n}$ such that $\pi_{n}>\alpha$, we note that

$$
\left(\pi_{n}-\alpha\right)^{2}=\pi_{n}-\pi_{n} \alpha-\alpha \pi_{n}+\alpha=\pi_{n}-\alpha
$$

so $\pi_{n}-\alpha$ is also a projection. For an appropriate choice of $\pi_{n}$ this is a natural candidate for the projection associated with the oppositely charged Dbrane. The physical motivation for 
this answer is based on the description of Dbranes as topological defects in a gauge bundle of higher dimensional branes [2]. We say that two Dbranes carry opposite charges if they are represented by the gauge bundles $E$ and $F$, such that the 'total' bundle $E \oplus F$ is isomorphic to a trivial bundle. It is easy to see that $\alpha$ and $\pi_{n}-\alpha$ satisfy the expected property provided that $\pi_{n}>\alpha$.

Now the condition for monodromy around $R_{c}$ is

$$
\alpha\left(\tau \mathrm{e}^{2 i \pi}\right)=U\left(\pi_{n}-\alpha(\tau)\right) U^{\dagger}
$$

where $|\tau|$ is the radius of a small circle around $R_{c}$ in the $z$ plane, see Fig. 1. Evaluating at $\tau=0$ we find a condition in the undeformed $K_{0}\left(\mathcal{A}_{0}\right)$ group:

$$
\alpha_{0}=U\left(\pi_{n}-\alpha_{0}\right) U^{\dagger}
$$

As a simple example, the reader may find it helpful to keep in mind the following twodimensional model:

$$
\alpha_{0}=\left(\begin{array}{ll}
1 & 0 \\
0 & 0
\end{array}\right), \quad \pi_{2}=\left(\begin{array}{ll}
1 & 0 \\
0 & 1
\end{array}\right), \quad U=\left(\begin{array}{ll}
0 & 1 \\
1 & 0
\end{array}\right) .
$$

where we assume that $U$ does not depend on $z$. This may be too strong an assumption in the general case, but suffices for the local model we construct.

It is clear that order by order one can reconstruct the solution to (2) and (8) as a power series in $\tau$ :

$$
\alpha(z)=\alpha_{0}+\tau \alpha_{1}+\tau^{2} \alpha_{2}+\ldots .
$$

The exact form of the solution will depend on the details of the $*$ product. To see this, we write down the first order equations that follow from (2) and (8):

$$
\left\{\alpha_{1}, U\right\}=0, \quad \alpha_{1}-\left\{\alpha_{1}, \alpha_{0}\right\}=\alpha_{0} *_{1} \alpha_{0}
$$

Proceeding in the same fashion, we expect to find solutions to (8) only at specific points on the moduli space (in our case, on the $z$ plane). These points represent the endpoints of the phase boundaries where some tachyonic modes become massless [7].

To summarize, in our local model, we have shown that when the equation (8) has a solution, there is a section of the $K$ group bundle with monodromy appropriate for describing the physics of a first order phase transition between different stable configurations of Dbranes. Thus $K$-theory combined with a knowledge of the deformation of the algebra product as a function of moduli (in this case the difference in the $B$ field flux through the 2-cycles) can be used to predict the phase structure of systems of (non-supersymmetric) Dbranes.

In conclusion we mention that there are other kinds of phase transitions when a sheaf $E$ associated with a Dbrane becomes unstable or split. The equation of the phase boundary follows from the Bogomolov condition:

$$
\int_{X}\left(2 r c_{2}-(r-1) c_{1}^{2}\right) \wedge J^{(n-2)}=0
$$

for a sheaf $E$ of rank $r$ over an $n$-dimensional space $X$ to be strictly stable with respect to a Kähler form $J$. 


\section{ACKNOWLEDGMENTS}

We have benefited from discussions with R. Bezrukavnikov, M. Kontsevich, J. Rosenberg, A. Schwarz, A. Sen, E. Sharpe and E. Witten. The work of S.G. was supported in part by grant RFBR No 98-02-16575 and Russian President's grant No 96-15-96939. The work of V.P. was supported in part by NSF grant PHY-9802484. 


\section{FIGURES}

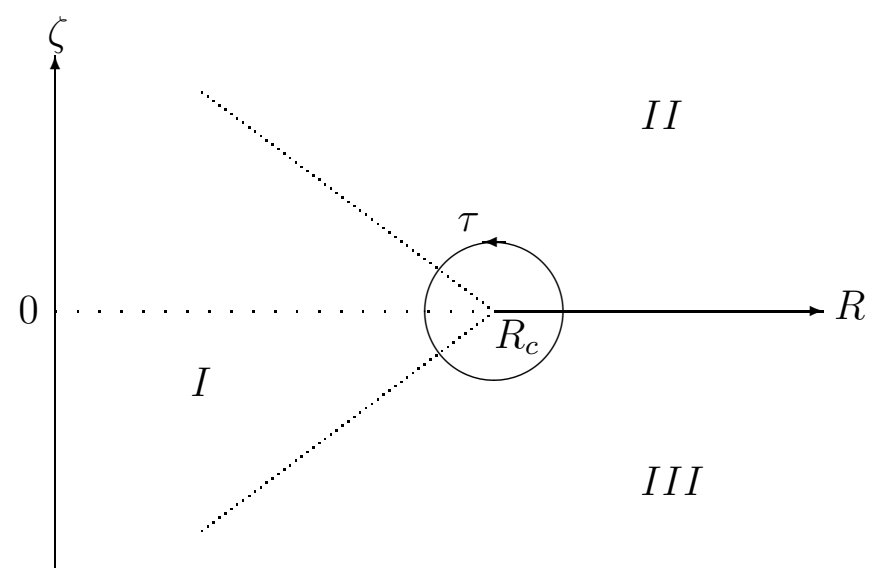

FIG. 1. Phase diagram in the $R-\zeta$ plane. 


\section{REFERENCES}

[1] A. Sen, J. High Energy Phys. 9806 (1998) 007; 9808 (1998) 010; 9808 (1998) 012; 9809 (1998) 023; 9810 (1998) 021.

[2] E. Witten, J. High Energy Phys. 9812 (1998) 019.

[3] R. Minasian, G. Moore, J. High Energy Phys. 9711 (1997) 002.

[4] A. Sen, "Non-BPS States and Branes in String Theory", hep-th/9904207.

[5] A. Lerda, R. Russo, "Stable non-BPS states in string theory: a pedagogical review", hep-th/9905006.

[6] K. Olsen, R. J. Szabo, "Constructing D-Branes from K-Theory", hep-th/9907140.

[7] J. Majumder, A. Sen, "Blowing up D-branes on Non-supersymmetric Cycles", hepth/9906109.

[8] A. Sen, J. High Energy Phys. 9812 (1998) 021

[9] J. Rosenberg, "Algebraic K-Theory and Its Applications", Springer-Verlag, 1991.

[10] B. Blackadar, "K-Theory for Operator Algebras", Cambridge Univ. Press, 1998.

[11] A. Connes, M. R. Douglas, A. Schwarz, J. High Energy Phys. 9802 (1998) 003.

[12] M. R. Douglas, C. Hull, J. High Energy Phys. 9802 (1998) 008; P.-M. Ho, Y.-S. Wu, Phys. Lett. B398 (1997) 52; C.-S. Chu, P.-M. Ho, Nucl. Phys. B550 (1999) 151; see also references in the "Two Lectures on D-Geometry and Noncommutative Geometry" by M. R. Douglas, hep-th/9901146.

[13] N. Seiberg and E.Witten, "String Theory and Noncommutative Geometry", hepth/9908142.

[14] V. Periwal, "Pointless strings", Princeton University dissertation (1988)

[15] B. Fedosov, J. Differential Geom. 40 (1994), 213.

[16] M. Kontsevich, "Deformation quantization of Poisson manifolds, I", q-alg/9709040.

[17] J. Rosenberg, "Behavior of $K$-theory under quantization", in Operator algebras and quantum field theory (Rome, 1996), 404.

[18] M. Pimsner and D. Voiculescu, J. Operator Theory 4 (1980) 93.

[19] S. Gukov, talk presented at the ASI Summer School Progress in String Theory and M-Theory, Cargèse, May 24 - June 5, 1999. 